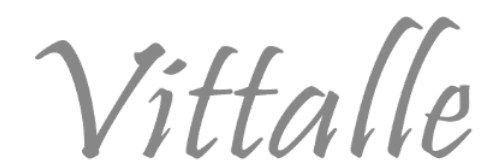

\title{
Comportamento dos poluentes do ar durante e após lockdown em uma cidade de médio porte
}

\author{
Ronan Adler Tavella ${ }^{\mathrm{a}, \mathrm{b}}$, Lucas da Silva Salimene ${ }^{\mathrm{b}}$, Sabrina Morales Ulguim ${ }^{\mathrm{b}}$, Romulo \\ Reginato Gabriel ${ }^{\mathrm{b}}$, Flavio Manoel Rodrigues da Silva Júnior ${ }^{\mathrm{a}} \mathrm{b}^{*}$
}

aPrograma de Pós-graduação em Ciências da Saúde, Faculdade de Medicina, Universidade Federal do Rio Grande FURG, Rio Grande, RS, Brasil

bLaboratório de Ensaios Farmacológicos e Toxicológicos, Instituto de Ciências Biológicas, Universidade Federal do Rio Grande - FURG, Rio Grande, RS, Brasil

\section{Histórico do Artigo}

Recebido em:

$02 / 11 / 2021$

Aceito em:

$29 / 11 / 2021$

Palavras-chave:

Poluição do ar;

pandemias, SARS-Cov-2;

Ozônio; Brasil

Keywords:

Air pollution; pandemics; SARS-Cov-2; Ozone;

Brazil

\begin{abstract}
RESUMO
A Pandemia da COVID-19 é, sem dúvida, o maior problema de saúde pública do século, até o momento. Entre as ações sancionadas pelos governos para conter a propagação do vírus estão as medidas de bloqueio e confinamento, conhecidas como lockdown. Vários locais do mundo mostraram melhoria da qualidade do ar decorrente da redução da mobilidade urbana durante os períodos de confinamento. No entanto, os resultados no Brasil ainda foram escassos. O objetivo do estudo foi avaliar o comportamento dos poluentes do ar em um lockdown de curta duração (64 horas) no município de Pelotas, RS. As concentrações de $\mathrm{O}_{3}, \mathrm{CO}, \mathrm{PM}_{2,5} \mathrm{NO}_{2}$ e $\mathrm{SO}_{2}$ foram medidas durante e após (até 30 horas) o lockdown por meio de dados de satélite e foram comparadas a média dos sete dias anteriores. Com exceção do $\mathrm{NO}_{2}$, todos os poluentes foram reduzidos durante o período do lockdown e os níveis mantidos mesmo após 30 horas do fim do bloqueio. A redução dos níveis de ozônio durante o lockdown é bastante questionada, em nível mundial, e parece estar relacionada a cidades menos populosas e com baixas concentrações de $\mathrm{NO}_{2}$. O estudo mostrou que um curto período de confinamento é suficiente para reduzir os níveis de poluentes no ar.
\end{abstract}

\section{Air pollutants behavior during and after lockdown in a medium-sized city}

\section{ABSTRACT}

The COVID-19 Pandemic is undoubtedly the greatest public health problem of the century, to date. Among the actions sanctioned by governments to curb the spread of the virus are blocking and confinement measures, known as lockdown. Several locations around the world have shown an improvement in air quality due to reduced urban mobility during periods of lockdown. However, results in Brazil are still scarce. The aim of the study was to evaluate the behavior of air pollutants in a short-term lockdown (64 hours) in the city of Pelotas, RS. The concentrations of $\mathrm{O}_{3}, \mathrm{CO}, \mathrm{PM}_{2.5} \mathrm{NO}_{2}$ and $\mathrm{SO}_{2}$ were measured during and after (up to 30 hours) the lockdown using satellite data and the average concentrations of the previous seven days were compared. With the exception of $\mathrm{NO}_{2}$, all pollutants were reduced during the lockdown period and levels maintained even 30 hours after its end. The reduction in ozone levels during the lockdown is widely questioned worldwide, and seems to be related to less populated cities and with low concentrations of $\mathrm{NO}_{2}$. The study showed that a short period of confinement is sufficient to reduce the levels of pollutants in the air.

\section{Introdução}

A Pandemia da COVID-19, doença causada pelo vírus denominado SARS-Cov-2, induziu governos de todo o mundo a adotarem medidas restritivas de mobilidade urbana e distanciamento social $(1,2)$. Embora o objetivo destas ações fosse a redução das taxas de infecção pelo vírus, as medidas adotadas trouxeram à tona outros temas, entre eles a questão ambiental (3). Entre estes tópicos, a redução dos níveis de poluentes atmosféricos

\footnotetext{
*Autor correspondente: f.m.r.silvajunior@gmail.com (Silva Júnior F.M.R.)
} 
tem sido objeto de inúmeros estudos ao redor do mundo (4). Estudos em diferentes regiões do mundo têm mostrado que as medidas de bloqueio ou confinamento são responsáveis pela redução de tráfego veicular e, em alguns casos, de atividade industrial, com uma consequente diminuição da emissão de poluentes para a atmosfera (5-13).

"Lockdown" tem sido o nome empregado para as medidas de confinamento adotadas pelos governos. Para a redução da mobilidade humana, os decretos ou leis municipais incluem ações que vão desde fechamento parcial ou total de comércios e serviços até impedir as pessoas de circularem nas ruas e avenidas das cidades $(1,2)$. No Brasil, o lockdown foi adotado em algumas cidades, tais como: São Luiz (MA), Fortaleza (CE) e Recife (PE). A duração do lockdown foi de nestas cidades foi entre 10 e 14 dias e incluía o fechamento total de comércios e serviços não-essenciais para manutenção da vida e da saúde, bem como o controle municipal do fluxo de pessoas e veículos $(14,15)$. Em agosto de 2020, Pelotas, uma cidade com aproximadamente 330 mil habitantes, situada no sudeste do Rio Grande do Sul, experimentou um lockdown com medidas mais restritivas e com duração de 64 horas. Além do controle do fluxo de pessoas e do fechamento de comércios e serviços não-essenciais, o Decreto Municipal fechou temporariamente serviços essenciais, tais como supermercados e agropecuárias (16).

Como mencionado anteriormente, a implementação de medidas de confinamento e distanciamento social mostraram ser efetivas para a redução de poluentes atmosféricos, especialmente para os poluentes dióxido de nitrogênio $\left(\mathrm{NO}_{2}\right)$, dióxido de enxofre $\left(\mathrm{SO}_{2}\right)$, partículas inaláveis finas $\left(\mathrm{PM}_{2,5}\right)$ e grossas $\left(\mathrm{PM}_{10}\right)$ e monóxido de carbono $(\mathrm{CO})$. Por outro lado, inúmeros estudos apontam o aumento na concentração de ozônio em grandes cidades causado pela adoção dessas medidas. Todos esses poluentes apresentam importância no cenário ambiental e de saúde humana. Além disso, a Organização Mundial de Saúde (OMS) em seu guia de qualidade do ar de 2021 reconhece que todos esses poluentes possuem impactos significativos na saúde de milhões de pessoas ao redor do mundo (17). Apesar da adoção dessas medidas ter impactado a concentração desses poluentes em diferentes localidades, os estudos em outras partes do mundo foram realizados observando períodos longos de lockdown, tais como em cidades da Europa $(9,10)$, China (13), Índia (8) e Estados Unidos da América (5). Assim, o objetivo do presente estudo foi avaliar a dinâmica dos poluentes atmosféricos após implementação do lockdown na cidade de Pelotas-RS, comparando com a média dos sete dias que antecederam a implementação do decreto municipal.

\section{Material e métodos}

\section{Area de estudo}

A região de estudo compreendeu dados da poluição atmosférica da cidade de pelotas, uma cidade com aproximadamente 330.000 habitantes, localizada na região sul do Brasil. Essa cidade inovou em sua região e, apesar de estar com medidas de distanciamento social por algumas semanas, experimentou um lockdown com duração de apenas 64 horas. Nessas condições, além de controlar o fluxo de pessoas e encerrar negócios e serviços não essenciais, o decreto municipal encerrou temporariamente serviços essenciais como supermercados, lojas agrícolas e postos de gasolina.

\section{Monitoramento dos poluentes atmosféricos}

Os níveis dos poluentes atmosféricos $\left(\mathrm{O}_{3}, \mathrm{NO}_{2}, \mathrm{SO}_{2}, \mathrm{PM}_{2,5}\right.$ e $\left.\mathrm{CO}\right)$ foram coletados de dados de satélites do Serviço de Monitoramento Atmosférico Copernicus da European Centre for Medium-Range Weather Forecasts (ECMWF), extraídos manualmente, em 
tempo real, a partir do aplicativo The Wealther Channel (IBM, EUA). A unidade de medida adotada para todos os poluentes foi $\mu \mathrm{g} / \mathrm{m}^{3}$.

O período de avaliação incluído no presente estudo foi entre 01 e 12 de agosto de 2020. Os dados diários entre 01 e 07 de agosto de 2020 foram utilizados a fim de obter a média dos sete dias que antecederam o lockdown. Para avaliação da dinâmica dos poluentes do ar durante o período do lockdown e pós-lockdown, oitenta e quatro registros foram coletados, iniciando 15 horas após o início do período compreendido pelo decreto municipal (11 h do dia 09 de agosto de 2020) e encerrando 30 horas após o final do lockdown (23 h do dia 12 de agosto de 2020). Para a elaboração dos gráficos foi utilizado o software GraphPad Prism 8.2.

\section{Resultados e discussão}

A Figura 1 apresenta a dinâmica temporal dos poluentes atmosféricos durante e após o período de lockdown na cidade de Pelotas, RS. O comportamento do ozônio e do monóxido de carbono foram similares durante o período avaliado, exibindo uma queda durante o lockdown e uma subsequente estabilização dos seus níveis em concentrações inferiores à média dos sete dias anteriores. Embora tenha apresentado picos acima da média histórica durante o período estudado, o comportamento do $\mathrm{PM}_{2,5}$ também foi de redução durante e após o lockdown, com uma estabilização em níveis bem abaixo da média dos sete dias anteriores. Diferente dos demais poluentes, o $\mathrm{NO}_{2}$ e $\mathrm{SO}_{2}$ tiveram picos diários que normalmente ultrapassavam a média histórica, ainda que a maior parte dos registros de $\mathrm{SO}_{2}$ foram abaixo desta média.
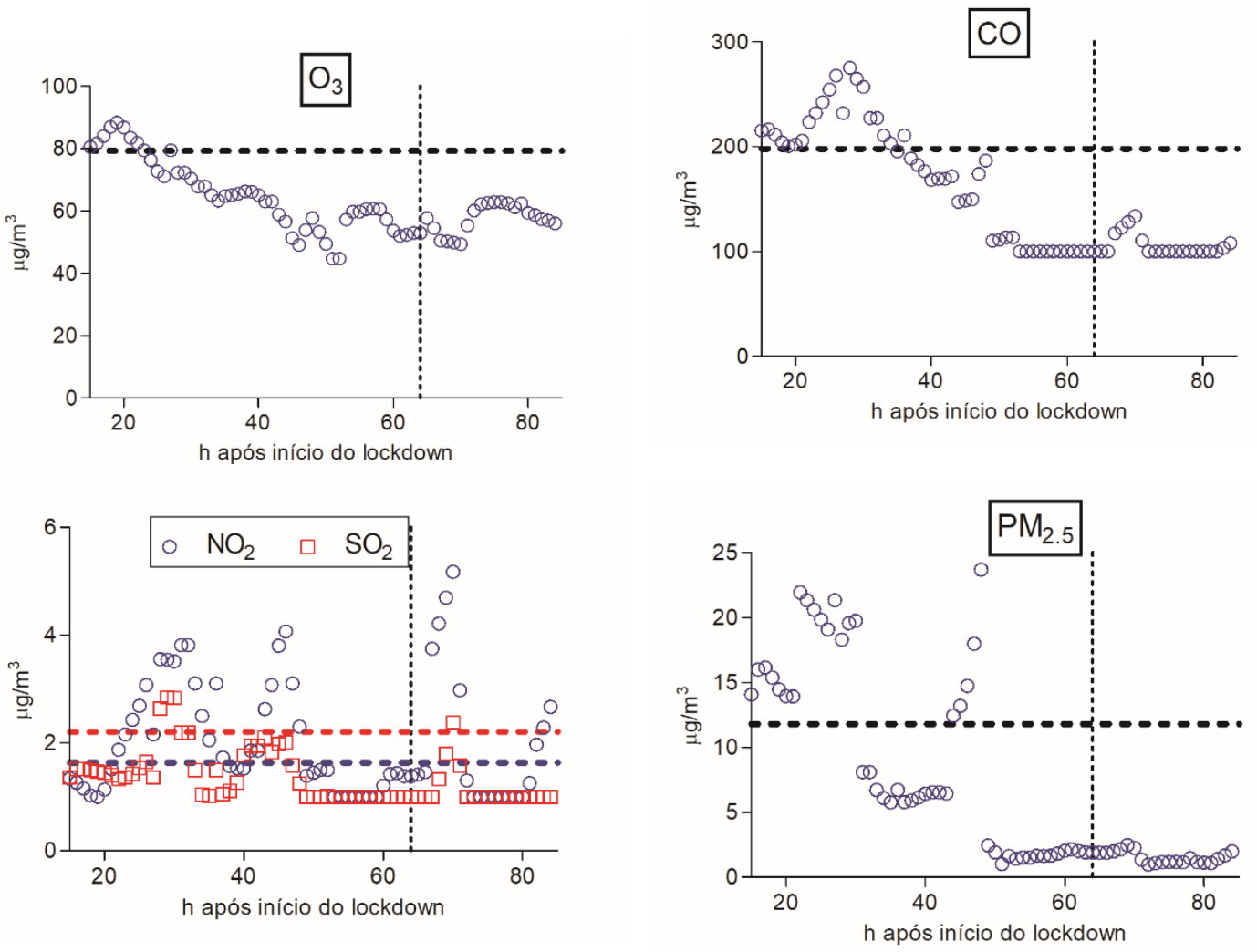

Figura 1 - Comportamento temporal dos poluentes atmosféricos durante e após o período de lockdown em Pelotas, RS, Brasil. A linha tracejada horizontal representa a média da concentração do poluente nos sete dias anteriores ao início do lockdown. A linha tracejada vertical indica o momento do término do lockdown. 
A Tabela 1 traz a média dos poluentes atmosféricos nos sete dias anteriores ao lockdown, durante e após o período de lockdown, bem como o percentual de registros que estiveram abaixo da média histórica no período compreendido durante e após o lockdown. Ozônio, monóxido de carbono, $\mathrm{PM}_{2,5}$ e dióxido de enxofre tiveram médias abaixo da média dos sete dias anteriores durante o período do lockdown e redução ainda maior no período de $30 \mathrm{~h}$ que compreendia o pós-lockdown. O percentual de registros abaixo da média histórica foi entre 70 e $90 \%$. Por outro lado, o dióxido de nitrogênio apresentou médias superiores à média dos sete dias anteriores, tanto no período do lockdown quanto no período pós-lockdown e um percentual de $54 \%$ dos registros foi inferior à média histórica para este poluente.

Tabela 1 - Média da concentração dos poluentes atmosféricos antes, durante e após o período de lockdown na cidade de Pelotas, RS, Brasil e percentual de valores registrados durante e após o lockdown abaixo da média dos setes dias anteriores

\begin{tabular}{cccccc}
\hline & $\mathrm{O}_{3}$ & $\mathrm{CO}$ & $\mathrm{PM}_{2.5}$ & $\mathrm{NO}_{2}$ & $\mathrm{SO}_{2}$ \\
\hline $\begin{array}{c}\text { Semana anterior } \\
\text { (média) }\end{array}$ & 79,3 & 197,8 & 11,8 & 1,6 & 2,1 \\
\hline $\begin{array}{c}\text { Durante lockdown } \\
\text { (média) }\end{array}$ & 67,2 & 191,6 & 11,5 & 2,2 & 1,6 \\
\hline $\begin{array}{c}\text { Após lockdown* } \\
\text { (média) }\end{array}$ & 57,2 & 104,2 & 1,6 & 1,8 & 1,1 \\
\hline $\begin{array}{c}\% \text { de valores abaixo } \\
\text { da média dos 7 dias } \\
\text { anteriores }\end{array}$ & $85,7 \%$ & $70 \%$ & $70 \%$ & $54,3 \%$ & $90 \%$ \\
\hline
\end{tabular}

*30 horas após o final do lockdown de 64h.

Esta redução dos níveis de poluentes em decorrência de medidas de confinamento também foi encontrada em outros estudos ao redor do mundo e também no Brasil (5-13, 18-20). O principal padrão de comportamento dos poluentes encontrado nestes estudos foi de diminuição nas concentrações de $\mathrm{NO}_{2}, \mathrm{PM}_{10}, \mathrm{PM}_{2,5}, \mathrm{SO}_{2}$ e $\mathrm{CO}$, e um consequente aumento nos níveis de $\mathrm{O}_{3}$. Entretanto, estes estudos foram realizados em grandes cidades e metrópoles, com elevada concentração populacional, assim esse comportamento pode ser dito característico a esta localidade, mas não para cidades de menor porte populacional. Além disso, até onde sabemos, este é o primeiro estudo que avaliou a dinâmica dos poluentes em um lockdown de curta duração e, mais ainda, revelando dados que esta medida foi efetiva para a redução dos níveis de poluentes. Os estudos chineses e europeus abordaram períodos iguais ou superiores a um mês de lockdown $(13,18)$, enquanto os estudos brasileiros investigaram períodos entre 30 e 90 dias de medidas de distanciamento social $(7,19,20)$. Essas reduções observadas em nosso estudo são positivas tanto para o meio ambiente da localidade avaliada como também para toda a saúde da população afetada. Entretanto, apesar de expressivas reduções, os níveis de poluentes observados na cidade de Pelotas não ultrapassam os níveis estabelecidos pela Organização Mundial da Saúde em seu guia de qualidade do ar (17).

Outra peculiaridade é que os estudos ao redor do mundo relataram diminuição de $\mathrm{NO}_{2}$ e um consequente aumento do $\mathrm{O}_{3}(7-11,13,18-20)$. Por outro lado, o presente estudo demonstrou redução dos níveis de $\mathrm{CO}, \mathrm{PM}_{2,5}$ e $\mathrm{SO}_{2}$, mas um comportamento atípico para $\mathrm{O}_{3}$ e $\mathrm{NO}_{2}$. O ozônio teve comportamento similar ao $\mathrm{CO}$, enquanto os níveis de $\mathrm{NO}_{2}$ estiveram durante boa parte do lockdown e do pós-lockdown acima da média histórica para o município. Embora a presença de $\mathrm{NO}_{2}$ esteja extremamente relacionada ao tráfego veicular e atividade industrial, os níveis deste poluente na cidade de Pelotas são bem inferiores aos demais locais do mundo onde foram realizados os estudos sobre a relação entre as medidas de confinamento e a redução dos níveis de poluentes. Esta pode ser uma 
razão para o período de lockdown no município não influenciar uma queda acentuada deste poluente. Importante ressaltar que as condições meteorológicas no período estudado foram uniformes, não apresentando alterações significativas, fazendo com que essa variável não influenciasse nos resultados observados.

Neste mesmo sentido, os estudos que tiveram reduções acentuadas nos níveis de $\mathrm{NO}_{2}$ (7-11,13,18-20) tiveram um aumento significativo nos níveis de $\mathrm{O}_{3}$ e os autores apontam que as elevadas concentrações de $\mathrm{NO}_{2}$ na atmosfera são importantes para a manutenção de níveis baixos de $\mathrm{O}_{3}$. Assim, nos cenários onde os níveis de $\mathrm{NO}_{2}$ foram reduzidos significativamente este aumento de ozônio é um comportamento esperado. Por outro lado, em regiões com baixas concentrações de $\mathrm{NO}_{2}$, este poluente pode influenciar pouco nos níveis de $\mathrm{O}_{3}$. Neste cenário, outros compostos ganham maior importância, tais como os compostos orgânicos voláteis (COVs) $(6,21,22)$. Esta é provavelmente a situação vivenciada no cenário do presente estudo. Isso acontece porque o ozônio troposférico é uma das espécies químicas mais complexas da atmosfera, sendo ele formado durante o dia por processos químicos não lineares a uma taxa que é determinada pelas concentrações atmosféricas de COVs e óxidos de nitrogênio. O comportamento aprofundado de formação do ozônio e como a dinâmica de achados científicos durante a pandemia de COVID-19 influenciou a observação desse poluente é encontrado em Tavella e Da Silva Júnior (22).

Por fim, outra situação peculiar do cenário estudado é a manutenção/redução dos níveis de poluentes atmosféricos mesmo após o fim do período de lockdown. Esta condição realça os benefícios ambientais e de saúde pública destas medidas de confinamento, atrelado à redução das atividades laborais e do tráfego de veículos automotores. Este realce está atrelado diretamente a informação disponibilizada pela OMS em seu guia sobre a qualidade do ar desenvolvido em 2021 (17), a qual afirma que entre sete a oito milhões de pessoas morrem todos os anos devido a poluição atmosférica. Assim, a redução apontada em nosso estudo, como efeito direto da mudança de hábitos da população de Pelotas, gera melhores condições ambientais e também de saúde para a população desta cidade.

Apesar dos múltiplos achados, nosso estudo apresenta algumas limitações. Primeiramente, nossos dados não foram comparados com dados históricos de monitoramento de poluentes atmosféricos de outros anos, ficando nossa comparação do período de lockdown limitada a um período no qual não havia lockdown mas havia medidas de distanciamento social impostas, talvez com isso amenizando o impacto nas reduções observadas. Além disso, não foram considerados a influência dos parâmetros meteorológicos na dinâmica dos poluentes. Ainda assim, a redução apontada no estudo aliada ao conhecimento de que a exposição humana aos poluentes atmosféricos está relacionada a uma série de problemas de saúde, incluindo doenças no sistema respiratório e cardiovascular (22), revela que a condição apresentada no estudo pode ser usada para uma reflexão sobre o uso excessivo de meios de transporte, sobretudo os automóveis e servir de subsídio para estudos de adequação dos níveis dos poluentes aos novos limites empregados pela PMS (17)

\section{Conclusão}

Com exceção do $\mathrm{NO}_{2}$, a concentração dos demais poluentes estudados reduziu durante o período de lockdown e os baixos níveis foram mantidos mesmo após $30 \mathrm{~h}$ do fim do período de confinamento. Para estes poluentes, um percentual entre 70 e $90 \%$ dos registros durante o período analisado estiveram abaixo da média dos sete dias anteriores ao início do lockdown. Assim, nosso estudo demonstrou que um lockdown curto, de 64 horas, é suficiente para implicar em elevadas reduções nos níveis de poluentes no ar. 


\section{Referências}

1. Bonaccorsi G, Pierri F, Cinelli M, Flori A, Galeazzi A, Porcelli F, et al. Economic and social consequences of human mobility restrictions under COVID-19. Proceedings of the National Academy of Sciences. 2020; 117(27): 15530-15535.

2. Lau H, Khosrawipour V, Kocbach P, Mikolajczyk A, Schubert J, Bania J, Khosrawipour T. The positive impact of lockdown in Wuhan on containing the COVID-19 outbreak in China. Journal of travel medicine. 2020; 27(3): taaa037.

3. Chakraborty I, Maity P. COVID-19 outbreak: Migration, effects on society, global environment and prevention. Science of the Total Environment. 2020; 728: 138882

4. Tavella RA, Da Silva-Júnior FMR. COVID-19 and air pollution: what do we know so far? VittalleRevista de Ciências da Saúde. 2020; 32(1): 22-31.

5. Berman JD, Ebisu K. Changes in U.S. air pollution during the COVID-19 pandemic. Science of the Total Environment. 2020; 739: 139864.

6. Tavella RA, Fernandes CLF, Penteado JO, De Lima Brum R, Florencio Ramires P, Coutelle Honscha L, Volcão LM, Dos Santos M, Muccillo-Baisch AL. Da Silva Júnior, FMR. Unexpected reduction in ozone levels in a mid-size city during COVID-19 lockdown. International Journal of Environmental Health Research, 2021; 1-14.

7. Nakada LYK, Urban RC. COVID-19 pandemic: Impacts on the air quality during the partial lockdown in São Paulo state, Brazil. Science of the Total Environment. 2020; 730: 139087.

8. Sharma S, Zhang M, Anshika, Gao J, Zhang H, Kota SH. Effect of restricted emissions during COVID19 on air quality in India. Science of the Total Environment. 2020; 728: 138878.

9. Sicard P, De Marco A, Agathokleous E, Feng Z, Xu X, Paoletti E, et al. Amplified ozone pollution in cities during the COVID-19 lockdown. Science of the Total Environment. 2020; 735: 139542.

10. Tobías A, Carnerero C, Reche C, Massagué J, Via M, Minguillón MC, et al. Changes in air quality during the lockdown in Barcelona (Spain) one month into the SARS-CoV-2 epidemic. Science of the Total Environment. 2020; 726: 138540.

11. Zambrano-Monserrate MA, Ruano MA. Has air quality improved in Ecuador during the COVID-19 pandemic? A parametric analysis. Air Quality, Atmosphere and Health. 2020; $1-10$.

12. Leão MLP, Penteado JO, Ulguim SM, Gabriel RR Dos Santos, M, Brum AN, Zhang L, da Silva Júnior, FMR. Health impact assessment of air pollutants during the COVID-19 pandemic in a Brazilian metropolis. Environmental Science and Pollution Research, 2021; 28(31): 41843-41850.

13. Chen K, Wang M, Huang C, Kinney PL, Anastas PT. Air pollution reduction and mortality benefit during the COVID-19 outbreak in China. The Lancet Planetary Health. 2020; 4(6): e210-e212.

14. Alves SMC, Ramos EMB, Delduque MC. Decretação de lockdown pela via judicial: medida (des) necessária? Cadernos de Saúde Pública. 2020; 36: e00116020.

15. Brasil, Estado do Maranhão. DECRETO No 35.7849 DE 03 DE MAIO DE 2020 [Internet]. 2020. Available from: https://sedihpop.ma.gov.br/files/2020/05/DECRETO-35.784-DE-3-DE-MAIO-DE2020.pdf

16. Brasil, Cidade de Pelotas, Estado do Rio Grande do Sul. DECRETO No 6.300, DE 05 DE AGOSTO DE 2020 [Internet]. 2020. Available from: http://leismunicipa.is/kryha

17. World Health Organization. WHO global air quality guidelines: particulate matter (PM2. 5 and PM10), ozone, nitrogen dioxide, sulfur dioxide and carbon monoxide: executive summary. 2021.

18. He G, Pan Y, Tanaka T. The short-term impacts of COVID-19 lockdown on urban air pollution in China. Nature Sustainability. 2020; 1-7.

19. Dantas G, Siciliano B, França BB, da Silva CM, Arbilla G. The impact of COVID-19 partial lockdown on the air quality of the city of Rio de Janeiro, Brazil. Science of the Total Environment. 2020; 729 : 139085.

20. Debone D, da Costa M, Miraglia S. 90 Days of COVID-19 Social Distancing and Its Impacts on Air Quality and Health in Sao Paulo, Brazil. Preprints 2020: 2020080022.

21. Kumar A, Singh D, Anandam K, Kumar K, Jain VK. Dynamic interaction of trace gases (VOCs, ozone, and NOx) in the rural atmosphere of sub-tropical India. Air Quality, Atmosphere \& Health. 2017; 10(7): 885-896.

22. Tavella RA, da Silva Júnior FMR. Watch out for trends: did ozone increased or decreased during the COVID-19 pandemic?. Environmental Science and Pollution Research, 2021; 1-6.

23. Cohen AJ, Brauer M, Burnett R, Anderson HR, Frostad J, Estep K, et al. Estimates and 25-year trends of the global burden of disease attributable to ambient air pollution: an analysis of data from the Global Burden of Diseases Study 2015. Lancet. 2017; 389: 1907e1918. 